\title{
Obesity Paradox in Cardiovascular Diseases and Research Progress
}

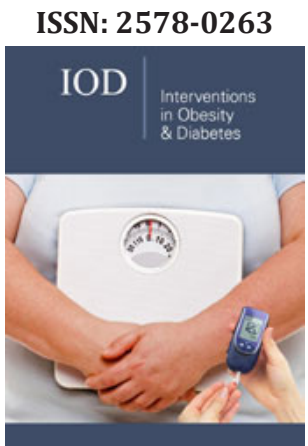

*Corresponding author: Zhong Chen, Department of Cardiology, Shanghai Jiao Tong University Affiliated Sixth People's Hospital. No. 300 Yishan Road, Shanghai 200233, P.R.China

Submission: 眥January 30, 2020

Published: 笽February 06, 2020

Volume 3 - Issue 3

How to cite this article: Changping $\mathrm{Li}$, Lili Zhang, Zhong Chen. Obesity Paradox in Cardiovascular Diseases and Research Progress. Interventions Obes Diabetes 3(3). IOD.000565.2020.

DOI: 10.31031/IOD.2020.03.000565

Copyright@ Zhong Chen. This article is distributed under the terms of the Creative Commons Attribution 4.0 International License, which permits unrestricted use and redistribution provided that the original author and source are credited.

\author{
Changping Li, Lili Zhang and Zhong Chen* \\ Department of Cardiology, Shanghai Jiao Tong University Affiliated Sixth People's Hospital, \\ No. 300 Yishan Road, Shanghai 200233, P.R.China
}

\begin{abstract}
In recent years, clinical evidence suggests that obese and overweight people with established cardiovascular diseases (CVDs) seem to have a better prognosis compared with lean patients. This phenomenon has been described as the 'obesity paradox', but there are also those who disagree with this seemingly deviant view, its existence remains a point of debate. This review tries to summarize the latest research progress of obesity paradox in CVDs and concludes with some explanations for this puzzling phenomenon.
\end{abstract}

Keywords: Overweight; Cardiovascular diseases; Hypertension; Obese patients

Abbreviations: CVDs: Cardiovascular Diseases; BMI: Body Mass Index; DBP: Diastolic Blood Pressure; WHR: Waist-to-Hip Ratio; CAD: Coronary Artery Disease; ACS: Acute Coronary Syndrome; AF: Atrial Fibrillation

\section{Introduction}

The World Health Organization has defined overweight and obesity based on body mass index (BMI; weight in kilograms/height in meters squared, $\mathrm{kg} / \mathrm{m}^{2}$ ). The prevalence of overweight and obesity as defined by BMI is rapidly increasing, particularly in children and adolescents, and associated with significantly increased risk of CVDs at an earlier age [1]. There is scientific consensus that obesity increases the risk of CVDs, excessive body weight associated with negative effects on hemodynamics, cardiac morphology and ventricular function, even metabolically healthy obese individuals had a higher risk for coronary heart disease $[2,3]$. It seems different from what we imagined, despite this negative correlation, recent epidemiological data and numerous studies found that overweight and obese people with established CVDs appear to have a better clinical prognosis. It is necessary for us to face up to this problem, because it may affect our diagnosis and treatment of patients. Is patients with CVDs fatter really fitter?

\section{Obesity paradox and hypertension}

Many epidemiological studies have confirmed the relationship between obesity and hypertension(HTN). Higher BMI were associated with higher risk of HTN in adults [4]. Barrett et al. [5] first reported the obesity paradox and HTN in 1985 in 1727 patients with systolic HTN, observing that obese patients with systolic blood pressure (SBP) $\geq 160 \mathrm{mmHg}$ had lower cardiovascular and ischemic heart disease mortality than nonobese patients with HTN. Subsequently, other scholars reported similar observations. Agarwal et al. [6] assessed 281,560 HTN emergency hospitalizations finding that overall mortality was significantly lower in-hospital mortality in those with obesity. In-hospital mortality has also been demonstrated to be lower in patients with pulmonary arterial HTN (PAH) and obesity (3.5\%) compared to the non-obese $(8.1 \%)$ from a 9-year nationwide study [7]. And found the combination of early marked weight loss and rapid blood pressure reduction seems to be harmful in obese elderly cardiovascular disease population [8]. Obesity, as defined by BMI, seems to have a positive effect in patients with hypertension. Whether the obesity paradox can be explained by a causal mechanism involving blood pressure(BP), Dorresteijn et al. [9] found each 5kg/ $\mathrm{m}^{2} \mathrm{BMI}$ was associated with $+3.8 \mathrm{mmHg}(95 \% \mathrm{CI} 3.0-4.6)$ SBP difference and $+2.3 \mathrm{mmHg}(95 \%$ CI 1.9-2.8) diastolic blood pressure(DBP) difference in both men and women. Measures of centralized adiposity compared with BMI were equally related with BP. In contrast, subcutaneous adipose tissue was only weakly related with DBP and not related with SBP and 
pulse pressure. Furthermore, $5 \mathrm{~kg} / \mathrm{m}^{2} \mathrm{BMI}$ change during follow-up was associated with $+8.1 / 5.1 \mathrm{mmHg}$ BP change. In a meta-analysis, Coutinho et al. [10] found that central obesity was associated with mortality (HR: 1.70, 95\% CI 1.58-1.83), whereas BMI was inversely associated with mortality (HR: $0.64,95 \%$ CI 0.59-0.69). Central obesity was also associated with higher mortality in the subset of subjects with normal BMI [11].

As stated above, more recent studies using a mix of more appropriate obesity indices, in particular central obesity, such as waist circumference (WC) and waist-to-hip ratio (WHR), skinfold thickness, and bioelectrical impedance, raise some doubt about the real significance of obesity paradox. And there are several studies that show either no protective effect of obesity or even worse effect [12]. Therefore, it is uncertain whether the obesity paradox is due to a real causal effect or to a statistical bias.

\section{Obesity paradox and coronary artery disease (CAD)}

Obesity is an independent risk factor for the development and progression of CAD. Obesity is associated with accelerated coronary atherosclerosis in adolescent and young adult men, atherosclerotic vascular lesions of patients with higher BMI values are more frequent and advanced compared to subjects with normal body weight [13]. Studies in the past few years have found the obesity paradox in $\mathrm{CAD}$, excess weight and obesity are associated with a favorable prognosis. A systematic review of 40 studies with 250,152 patients show that patients with a low BMI had an increased relative risk (RR) for total mortality (RR=1.37 [95\% CI 1.32-1.43] ), and cardiovascular mortality (1.45 [1.16-1.81]) compared with those for people with a normal BMI [14]. If so, is fatter healthier? One research studied 3,307 individuals (1,038 women) with CAD, after adjusted for age, smoking, blood pressure, diabetes, alcohol, and self-reported health, observed that no mortality risk reductions associated with weight loss in individuals with CAD, and reduced mortality risk associated with weight gain in individuals who were normal weight at baseline [15]. The result seems optimistic. The latest research seems to offer different views, a large meta-analysis of 89 studies including 1.3 million CHD patients also confirmed an obesity paradox, which was evident during early follow-up even in patients with severe obesity. Such protective effects, however, seemed to disappear after approximately 5 years [16]. Younis et al. [17] studied 15,357 patients with stable CAD, Kaplan-Meier survival analysis showed that at 20 years of follow-up the rate of all-cause mortality was significantly higher among obese patients (67\%) compared to overweight (61\%) and normal weight (61\%). Data from other research shows that there is no obesity paradox when measuring body fat percentage (BF\%) instead of BMI $[18,19]$.

The author think underweight patients tend to be more malnourished and cachectic and have a higher prevalence of comorbid conditions such as malignancy and heart failure. In addition, underweight patients tend to be older. They also suggest that there may be a lead-time bias, in that patients with elevated BMI are investigated and treated at an earlier stage in the disease process. Although they found an increased prevalence of other cardiovascular risk factors such as diabetes and hypertension in patients with elevated BMI, the reduced risk was independent of these comorbid conditions, and its association with increased BMI was not attenuated by their inclusion in the model.

\section{Obesity paradox and acute coronary syndrome(ACS)}

A meta-analysis of 26 observational studies, including nearly 218,532 patients by Niedziela et al. [20] showed that the highest risk of mortality was found in low BMI patients (RR 1.47[95 \% CI 1.24-1.74]), while overweight, obese and severely obese patients had lower mortality compared with those with normal BMI. In one study, Kouvari et al. [21] have demonstrated that BMI status and 10-year ACS prognosis followed a J-shape association ( $\mathrm{p}=0.009)$. Overweight patients had significantly better ACS prognosis than their normal-weight counterparts (OR=0.45, 95\% CI $(0.23,0.90)$ ). The aforementioned paradoxical association was retained only in patients with specific conditions. In a large and unselected group of patients with ACS, the relation between BMI and mortality was U-shaped and was also observed in patients with type 2 diabetes mellitus and ACS, the protective effect of obesity disappeared in patients treated with insulin [22,23]. Migaj et al. [24]reported that obesity seems to have a different influence on outcomes in both genders, only male patients seem to contribute to the obesity paradox observed in patients with ACS. Diercks et al. [25] showed that overweight or obese were younger and more likely to present with comorbid conditions, including diabetes mellitus, hypertension, and hyperlipidemia. Although obesity appears to be a risk factor for developing ACS at a younger age, it also appears to be associated with more aggressive ACS management and, ultimately, improved outcomes. Age, lifestyle, and fitness are also variables that must be considered, as well as preexisting diseases. Sex differences could also be important, as observed in a study from the Cleveland Clinic, in which BMI has a different impact on mortality in males versus females and obesity paradox disappears in males after adjustment for potential confounders [26]. The protective role of overweight and obesity against all-cause mortality seems to be linked to a condition of high fitness. Also in older subjects (60 or over), physical fitness may be a more important determinant of survival than overweight [27].

In patients presenting with ST-segment elevation myocardial infarction (STEMI) and left ventricle dysfunction, with worse outcomes for those with normal weight, when compared to overweight or obese individuals [28]. These findings are consistent with the obesity-paradox. In AMI patients with primary percutaneous coronary intervention (PCI), but the obesity paradox was recognized only in patients in the elderly age group and not in the younger age group, which means the prognostic impact of BMI may differ by age in AMI patients [29,30].

With regards to the impact of BMI on survival after coronary artery bypass graft (CABG), the results are also inconsistent. Takagi et al. [31] reported that overweight, but not obesity, may be associated with better short-term and mid-to-long-term postCABG survival compared to normal weight. A 2014 analysis of 
12 CABG studies in 60,000 patients found worst survival rates in underweight patients, who had a 2.7 -fold higher mortality than patients with normal BMI [32]. But there was a tendency to lower survival among patients with BMI $>35 \mathrm{~kg} / \mathrm{m}^{2}$ [33]. Another study observed that there is no significant difference in hospital or follow-up mortality among patients undergoing CABG surgery when modified by BMI, obese patients gained less benefit in terms of QoL dimension, and there was no significant difference in overall mortality in the long-term follow-up [34].

\section{Obesity paradox and atrial fibrillation(AF)}

Obesity is an independent risk factor for $\mathrm{AF}$ and may also be a risk factor for progression of paroxysmal to persistent $\mathrm{AF}$, which carries higher morbidity and mortality [35,36]. In 2009, Lavic et al. [37]found obesity paradox exists in patients with AF with overweight and obese patients have a better prognosis than leaner patients with the same degree of severity of cardiovascular disease/AF. In patients with CHD and heart failure, overweight and obese patients with AF have a considerably better prognosis than do those patients with normal BMI. In a study of 431, 734 hospitalizations for AF, Agarwal et al. [38] found obese patients had lower risk-adjusted odds of in-hospital mortality and stroke events. In contrast, the obese group had a better prognosis in major adverse events compared with the normal weight group (HR 0.34, 95\% CI 0.13-0.89, $\mathrm{p}=0.029$ ). This paradox also exists in the elderly $\mathrm{AF}$ patients and in patients with AF treated with oral anticoagulants $[39,40]$.

But the evidence from the INTERHEART study, which assessed 2,540 patients enrolled in the EORP-AF Registry with 1 year followup, finding all-cause mortality was significantly different according to BMI among female patients (9.3\% normal BMI, 5.3\% overweight, and $4.3 \%$ obese, $\mathrm{P}=0.023$ ), but not among male patients ( $\mathrm{P}=0.748)$. The composite outcome of thromboembolic events and death was also significantly different, being lower in obese females ( $\mathrm{P}=0.035)$. Among male patients, bleeding events were significantly more frequent in obese subjects $(\mathrm{P}=0.035)$ [41]. A meta-analysis of 6 observational studies, including nearly 2358 patients by Guijian et al. [42] showed that elevated BMI increased the risk of $\mathrm{AF}$ recurrence compared to normal BMI by $31 \%$.

\section{Obesity paradox and heart failure(HF)}

Previous research found that obesity may result in HF by inducing changes in cardiac hemodynamics, structure, function and conduction. Recent years, there is a large amount of evidence which suggests there is obesity paradox in patients with heart failure, risk for total mortality and cardiovascular mortality and hospitalization was highest in patients with chronic HF who were underweight as defined by low BMI, whereas risk for cardiovascular mortality and hospitalization was lowest in overweight subjects [43]. In a recent meta-analysis of nine observational studies including heart failure patients, Oreopoulos et al. [44] compared all-cause mortality with BMI and found that overweight and obese patients had a more favorable prognosis than those who were either underweight or normal weight. Weight loss $\geq 5 \%$ in patients with chronic HF was associated with high long-term mortality, particularly among obese patients with HF [45]. In a study of 6,142 patients with acute decompensated HF, Shah et al. [46] also found the obesity paradox, but the "protective" association of BMI with mortality was confined to persons with older age, decreased cardiac function, no diabetes and de novo HF. Habbu et al. [47] pointed towards a U-shaped relationship between BMI, and survival in heart failure patients, with poor survival in both cachectic patients and patients with severe obesity.

The author think heavier patients may be presenting earlier with worse symptoms but less advanced disease state than patients with lower BMI. Patients with higher BMI were less likely to have systolic failure than patients with lower BMI. Patients with healthy weight may not have adequate nutritional stores or metabolic reserves to overcome the metabolic demands and catabolic stress resulting from an acute exacerbation of heart failure. The obesity paradox may be driven by deleterious effects of cachexia, not salutary effects of obesity. Furthermore, the survival paradox of BMI disappears also in diabetic patients with heart failure [48] or when peak oxygen consumption $\left(\mathrm{VO}_{2}\right)$ was used for multivariate analysis [49]. These results support the superior prognostic power of peak oxygen consumption and diabetes compared to obesity, which attenuates the "obesity paradox" phenomenon [50].

\section{Obesity paradox and stroke}

Many research found that overweight and obesity were associated with progressively increasing risk of ischemic stroke [51]. What is surprising that most observational data indicate a survival benefit of obese patients after stroke $[52,53]$. In a Korean observational study, including 34,132 patients with acute ischemic stroke by Kim et al. showed that compare to normal body weight, mortality risk was lowest in overweight (HR 0.77 95\% CI $0.63-$ 0.93 ) followed by the obese (HR $0.83,95 \%$ CI $0.74-0.92$ ), whereas highest in under-weight patients (HR 1.36, 95\% CI 1.25-1.48) [54]. This is supported by evidence from the Andersen et al. [55] which assessed 45615 acute first-ever stroke patients, finding that mortality was significantly lower in overweight (HR 0.72; 95\% CI 0.68-0.78) and obese (HR 0.80; 95\% CI 0.73-0.88) patients while significantly higher in underweight patients (HR 1.66; 95\% CI 1.49-1.84) compared with normal weight patients. Subsequently, Wohlfahrt et al. [56] found normal weight at hospital admission and weight loss after ischemic stroke are independently associated with increased mortality. Though more observational data indicate a survival benefit of obese patients after stroke, but methodological concerns still exist. Among which no obesity paradox was observed in patients after intravenous thrombolysis [57].

The author think there is a need for well-designed randomized controlled trials assessing the effects of weight reduction on stroke risk in obese patients. In summary, most observational data indicate a survival benefit of obese patients after stroke, but a number of methodological concerns exist. Available data support obesity as an independent risk factor for occurrence of stroke, and weight reduction in overweight or obese patients is still recommended for primary stroke prevention. 


\section{Other Issues}

\section{When assessing obesity in different ways}

Previous studies have relied predominantly on the BMI to assess the association of adiposity with the risk of death, but few have examined whether the other methods for evaluating obesity contributes to the prediction of death. More recent studies have investigated obesity paradox in patients not only on the basis of body weight, but also with the use of other measures of body fat, such as WC, WHR, skinfold thickness, and bioelectrical impedance, and the results show a picture much less clear [58]. In the study by Pischon et al. [59], BMI remained significantly associated with the risk of death in models that included WC or WHR $(\mathrm{P}<0.001)$. This supports that both general adiposity and abdominal adiposity are associated with the risk of death and support the use of WC or WHR in addition to BMI in assessing the risk of death. Another opinion is that the obesity paradox is mainly due to the effect of confounding on BMI and disappears on other adiposity measures as waist, WHR and $\mathrm{BF} \%$ [60].

\section{When considering metabolism}

Metabolically healthy obese phenotype (MHO) refers to obese individuals without metabolic abnormalities such as dyslipidemia, insulin resistance or hypertension. A meta-analysis aiming to assess the risks of cardiovascular events and all-cause mortality for MHO individuals and confirms a positive association between MHO and the risk of cardiovascular events [61]. The latest study used non-targeted metabolomics and whole-genome sequencing to identify metabolic and genetic signatures of obesity. An abnormal metabolome associated with a 2 - to 5 -fold increase in cardiovascular events when comparing individuals who were matched for BMI but had opposing metabolome signatures. The health consequences observed across the various BMI groups indicate that there is a durable benefit of maintaining a healthy metabolome signature and point to an ongoing risk for the individuals that have an unhealthy metabolome despite stability of BMI [62]. A recent study of 54089 men and women from five cohort studies found that obesity may not be associated with higher risk for all-cause mortality compared to lean healthy individuals in the absence of metabolic abnormalities [63].

\section{When rethinking the $\mathrm{BMI}$}

BMI is sometimes criticized for not distinguishing fat from lean mass and ignoring fat distribution, leaving its ability to detect health effects unclear [64,65]. Obesity defined by BMI alone is a remarkably heterogeneous condition with varying cardiovascular and metabolic manifestations among individuals. Although it is clear that the accumulation of visceral/ectopic fat is a major contributor to cardiovascular and metabolic risk above and beyond BMI, implementation of fat distribution assessment into clinical practice remains a challenge. This paradox may also arise from BMI failing to measure fat redistribution to a centralized position in later life. The claimed BMI-defined overweight risk paradox may result in part from failing to account for central adiposity, rather than reflecting a protective physiologic effect of higher body-fat content in later life [66]. At present, the definition of obesity is not ideal. BMI is not as good as body fat, fat distribution, body weight and body fluid components. These controversial effects of obesity are mostly due to the use of different indices of obesity in various studies.

\section{The other possible explanations for this phenomenon}

It is well known that excess weight and obesity, as phenomenon of the metabolic syndrome, lead to enhanced cardiovascular risk, endothelial dysfunction, inflammation, and atherosclerosis. The analyses show that in the case of $2 \%$ of thin patients, comorbid conditions, mostly malignant diseases, heart failure, malnutrition or multiple organ dysfunction could be observed. Moreover, these patients were much older than their normal weight or obese counterparts [67]. Adiponectin, a secretory protein produced by adipocytes and inversely proportional to BMI, is a possible mediator for the so-called "obesity paradox" [68].

\section{Conclusion}

Obesity has reached epidemic dimensions worldwide as a major risk factor for the metabolic syndrome, diabetes mellitus, dyslipidemia and hypertension, all significant causes of CVDs. When comes to the 'obesity paradox', inherent limitations of BMI as an index of adiposity, as well as methodological biases and the presence of confounding factors, may account for the observed findings of clinical studies, but also may distort the true relationship between obesity and mortality. Obese individuals generally present earlier, so disease states may be recognized and treated earlier. The obesity paradox may just be an over representation of cachexia. Therefore, it is possible that observations supporting the existence of an obesity paradox could be driven by both the limitations of BMI as an obesity index and clinical studies per se and may represent an epiphenomenon rather than a true causal relationship.

\section{Acknowledgement}

The study was supported by Science and Technology Development Fund of Shanghai Pudong New Area (PKJ2018-Y53) awarded in 2019 and the 2017 Shanghai Hospital Development Center Research Project (SHDC12017X24). The manuscript was written without the role from funders concerning study design, data collection, preparation and publish of the manuscript.

\section{References}

1. Khan SS, Ning H, Wilkins JT, Allen N, Carnethon M, et al. (2018) Association of body mass index with lifetime risk of cardiovascular disease and compression of morbidity. JAMA Cardiology 3(4): 280-287.

2. Alpert M A, Kamalesh K, Obai A, Rugheed G (2018) Obesity and cardiac remodeling in adults: Mechanisms and clinical implications. Prog Cardiovasc Dis 61(2): 114-123.

3. Caleyachetty R, Thomas GN, Toulis KA, Mohammed N, Gokhale KM, et al. (2017) Metabolically healthy obese and incident cardiovascular disease events among 3.5 million men and women. J Am Coll Cardiol 70(12): 1429-1437.

4. Crump C, Sundquist J, Winkleby MA, Sundquist K (2016) Interactive effects of physical fitness and body mass index on the risk of hypertension. JAMA Intern Med 176(2): 210-216. 
5. Barrett Connor E, Khaw KT (1985) Is hypertension more benign when associated with obesity? Circulation 72(1): 53-60.

6. Agarwal MA, Shah M, Garg L, Lavie CJ (2018) Relationship between obesity and survival in patients hospitalized for hypertensive emergency. Mayo Clin Proc 93(2): 263-265.

7. Agarwal M, Agrawal S, Garg L, Lavie CJ (2017) Relation between obesity and survival in patients hospitalized for pulmonary arterial hypertension (from a Nationwide inpatient sample database 2003 to 2011). Am J Cardiol 120(3): 489-493.

8. Seimon RV, Espinoza D, Ivers L, Gebski V, Finer N, et al. (2014) Changes in body weight and blood pressure: Paradoxical outcome events in overweight and obese subjects with cardiovascular disease. Int J Obes (Lond) 38(9): 1165-1171.

9. Dorresteijn JA, Spiering W, Van Der Graaf Y, Visseren FLJ (2012) Relation between adiposity and hypertension persists after onset of clinically manifest arterial disease. J Hypertens 30(12): 2331-2337.

10. Coutinho T, Goel K, Correa de Sa D, Kragelund C, Kanaya AM, et al. (2011) Central obesity and survival in subjects with coronary artery disease: A systematic review of the literature and collaborative analysis with individual subject data. J Am Coll Cardiol 57(19): 1877-1886.

11. Sahakyan KR, Somers VK, Rodriguez Escudero JP, Hodge DO, Carter RE, et al. (2015) Normal-weight central obesity: Implications for total and cardiovascular mortality. Ann Intern Med 163(11): 827-835.

12. Chrysant SG, Chrysant GS (2013) New insights into the true nature of the obesity paradox and the lower cardiovascular risk. J Am Soc Hypertens $7(1): 85-94$.

13. Bowman K, Atkins JL, Delgado J, Kos K, Kuchel GA, et al. (2017) Central adiposity and the overweight risk paradox in aging: Follow-up of 130,473 UK biobank participants. Am J Clin Nutr 106(1): 130-135.

14. Romero Corral A, Montori VM, Somers VK, Korinek J, Thomas RJ, et al. (2006) Association of bodyweight with total mortality and with cardiovascular events in coronary artery disease: A systematic review of cohort studies. Lancet 368(9536): 666-678.

15. Moholdt T, Lavie CJ, Nauman J (2018) Sustained physical activity, not wight loss, associated with improved survival in coronary heart disease. J Am Coll Cardiol 71(10): 1094-1101.

16. Wang ZJ, Zhou YJ, Galper BZ, Gao F, Yeh RW, et al. (2015) Association of body mass index with mortality and cardiovascular events for patients with coronary artery disease: A systematic review and meta-analysis. Heart 101(20): 1631-1638.

17. Younis A, Younis A, Goldkorn R, Goldenberg I, Peled Y, et al. (2018) The association of body mass index and 20-year all-cause mortality among patients with stable coronary artery disease. Heart, Lung and Circulation 28(5): 719-726

18. Medina Inojosa JR, Somers VK, Thomas RJ, Jean N, Jenkins SM, et al. (2018) Association between adiposity and lean mass with long-term cardiovascular events in patients with coronary artery disease: No paradox. J Am Heart Assoc 7(10): e007505.

19. Medina Inojosa JR, Batsis JA, Supervia M, Somers VK, Thomas RJ, et al. (2018) Relation of waist-hip ratio to long-term cardiovascular events in patients with coronary artery disease. Am J Cardiol 121(8): 903-909.

20. Niedziela J, Hudzik B, Niedziela N, Gąsior M, Gierlotka M, et al. (2014) The obesity paradox in acute coronary syndrome: A meta-analysis. Eur J Epidemiol 29(11): 801-812.

21. Kouvari M, Chrysohoou C, Tsiamis E, Kosyfa H, Kalogirou L, et al. (2017) The overweight paradox in the prognosis of acute coronary syndrome for patients with heart failure-A truth for all? A 10-year follow-up study. Maturitas 102: 6-12.
22. Angeras 0, Albertsson P, Karason K, Ramunddal T, Matejka G, et al. (2013) Evidence for obesity paradox in patients with acute coronary syndromes: A report from the Swedish coronary angiography and angioplasty registry. Eur Heart J 34(5): 345-53.

23. Jong CB, Li HY, Pan SL, Hsieh MY, Su FY, et al. (2019) Relationship between body mass index, antidiabetic agents, and midterm mortality in patients with both type 2 diabetes mellitus and acute coronary syndrome. J Am Heart Assoc 8(7): e011215.

24. Migaj J, Prokop E, Straburzyńska Migaj E, Lesiak M, Grajek S, et al. (2015) Does the influence of obesity on prognosis differ in men and women? A study of obesity paradox in patients with acute coronary syndrome. Kardiol Pol 73(9): 761-767.

25. Diercks DB, Roe MT, Mulgund J, Pollack CV, Kirk JD, et al. (2006) The obesity paradox in non-ST-segment elevation acute coronary syndromes: Results from the can rapid risk stratification of unstable angina patients suppress adverse outcomes with early implementation of the American College of Cardiology/American Heart Association guidelines quality improvement initiative. Am Heart J 152(1): 140-148.

26. Vest AR, Wu Y, Hachamovitch R, Young JB, Cho L (2015) The heart failure overweight/obesity survival paradox: The missing sex link. JACC Heart Fail 3(11): 917-926.

27. Yerrakalva D, Mullis R, Mant J (2015) The associations of fatness, fitness, and physical activity with all-cause mortality in older adults: A systematic review. Obesity 23(10): 1944-1956.

28. Samanta R, Pouliopoulos J, Kumar S, Kumar S, Narayan A, et al. (2018) Influence of BMI on inducible ventricular tachycardia and mortality in patients with myocardial infarction and left ventricular dysfunction: The obesity paradox. Int J Cardiol 265: 148-154.

29. Fukuoka S, Kurita T, Dohi K, Masuda J, Seko T, et al. (2019) Untangling the obesity paradox in patients with acute myocardial infarction after primary percutaneous coronary intervention (detail analysis by age). Int J Cardiol 289: 12-18.

30. Bucholz EM, Beckman AL, Krumholz HA, Krumholz HM (2016) Excess weight and life expectancy after acute myocardial infarction: The obesity paradox reexamined. Am Heart J 172: 173-181.

31. Takagi H, Umemoto T (2016) Overweight, but not obesity, paradox on mortality following coronary artery bypass grafting. J Cardiol 68(3): 215-221.

32. Sharma A, Vallakati A, Einstein AJ, Lavie CJ, Arbab Zadeh A, et al. (2014) Relationship of body mass index with total mortality, cardiovascular mortality, and myocardial infarction after coronary revascularization: Evidence from a meta-analysis. Mayo Clin Proc 89(8): 1080-1100.

33. Efros LA, Samorodskaya IV (2015) Survival of overweight patients after coronary artery bypass surgery. Does the obesity paradox play a role? Kardiologiia 55(7): 45-50.

34. Hokkanen M, Jarvinen O, Huhtala H, Laurikka J (2018) The effect of obesity on long-term survival and health-related quality of life after coronary artery bypass grafting. Coronary Artery Disease 29(5): 378383.

35. Lavie CJ, Pandey A, Lau DH, Alpert MA, Sanders P (2017) Obesity and atrial fibrillation prevalence, pathogenesis, and prognosis: Effects of weight loss and exercise. J Am Coll Cardiol 70(16): 2022-2035.

36. Lavie CJ, De Schutter A, Parto P, Jahangir E, Kokkinos P, et al. (2016) Obesity and prevalence of cardiovascular diseases and prognosis-the obesity paradox updated. Prog Cardiovasc Dis 58(5): 537-547.

37. Lavic CJ, Milani RV, Ventura HO (2009) Obesity and cardiovascular disease risk factor, paradox, and impact of weight loss. J Am Coll Cardiol 53(21): 1925-1932. 
38. Agarwal MA, Garg L, Shah M, Patel B, Jain N, et al. (2019) Relation of obesity to outcomes of hospitalizations for atrial fibrillation. Am J Cardiol 123(9): 1448-1452.

39. Yanagisawa S, Inden Y, Yoshida N, Kato H, Miyoshi FA, et al. (2016) Body mass index is associated with prognosis in Japanese elderly patients with atrial fibrillation: An observational study from the outpatient clinic. Heart Vessels 31(9): 1553-1561.

40. Sandhu RK, Ezekowitz J, Andersson U, Alexander JH, Granger CB, et al. (2016) The obesity paradox in atrial fibrillation: Observations from the ARISTOTLE (Apixaban for Reduction in Stroke and Other Thromboembolic Events in Atrial Fibrillation) trial. Eur Heart J 37 (38): 2869-2878.

41. Boriani G, Laroche C, Diemberger I, Fantecchi E, Meeder J, et al. (2018) Overweight and obesity in patients with atrial fibrillation: Sex differences in 1-year outcomes in the EORP-AF general pilot registry. J Cardiovasc Electrophysiol 29(4): 566-572.

42. Guijian L, Jinchuan Y, Rongzeng D, Jun Q, Jun W, et al. (2013) Impact of body mass index on atrial fibrillation recurrence: A meta-analysis of observational studies. Pacing Clin Electrophysiol 36(6): 748-56.

43. Sharma A, Lavie CJ, Borer JS, Vallakati A, Goel S, et al. (2015) Metaanalysis of the relation of body mass index to all-cause and cardiovascular mortality and hospitalization in patients with chronic heart failure. Am J Cardiol 115(10):1428-1434.

44. Oreopoulos A, Padwal R, Kalantar zadeh K, Fonarow GC, Norris CM, et al. (2008) Body mass index and mortality in heart failure: A meta-analysis. Am Heart J 156(1): 13-22.

45. Zamora E, Díez Lopez C, Lupon J, de Antonio M, Domingo M, Santesmases J, et al. (2016) Weight loss in obese patients with heart failure. J Am Heart Assoc 5(3): e002468.

46. Shah R, Gayat E, Januzzi JL, Sato N, Cohen Solal A, et al. (2014) Body mass index and mortality in acutely decompensated heart failure across the world: A global obesity paradox. J Am Coll Cardiol 63(8): 778-785.

47. Habbu A, Lakkis NM, Dokainish H (2005) The obesity paradox: Fact or fiction? Am J Cardiol 98(7): 944-948.

48. Zamora E, Lupón J, Enjuanes C, Pascual Figal D, de Antonio M, et al. (2016) No benefit from the obesity paradox for diabetic patients with heart failure. Eur J Heart Fail 18(17): 851-858.

49. Piepoli MF, Corrà U, Veglia F, Bonomi A, Salvioni E, et al. (2016) Exercise tolerance can explain the obesity paradox in patients with systolic heart failure: Data from the MECKI Score Research Group. Eur J Heart Fail 18(5): 545-553.

50. Piepoli MF (2017) Obesity in heart failure: Is it time to rethink the paradox? Eur J Heart Fail 19(12): 1736.

51. Yatsuya H, Folsom AR, Yamagishi K, North KE, Brancati FL, et al. (2010) Race-and sex-specific associations of obesity measures with ischemic stroke incidence in the Atherosclerosis Risk in Communities (ARIC) study. Stroke 41(3): 417-425.

52. Aparicio HJ, Himali JJ, Beiser AS, Davis Plourde KL, Vasan RS, et al. (2017) Overweight, obesity, and survival after stroke in the framingham heart study. J Am Heart Assoc 6(6): e004721.

53. Park H, Lee HW, Yoo J, Lee HS, Nam HS, et al. (2019) Body mass index and prognosis in ischemic stroke patients with type 2 diabetes mellitus. Front Neurol 10: 563.
54. Kim BJ, Lee SH, Jung KH, Yu KH, Lee BC, et al. (2012) Dynamics of obesity paradox after stroke, related to time from onset, age, and causes of death. Neurology 79(9): 856-863.

55. Andersen KK, Olsen TS (2015) The obesity paradox in stroke: Lower mortality and lower risk of readmission for recurrent stroke in obese stroke patients. Int J Stroke 10(1): 99-104.

56. Wohlfahrt P, Lopez Jimenez F, Krajcoviechova A, Jozifova M, Mayer O, et al. (2015) The obesity paradox and survivors of ischemic stroke. J Stroke Cerebrovasc Dis 24(6): 1443-1450.

57. Oesch L, Tatlisumak T, Arnold M, Sarikaya H (2017) Obesity paradox in stroke-Myth or reality? A systematic review. PLoS One 12(3): e0171334.

58. Gupta PP, Fonarow GC, Horwich TB (2015) Obesity and the obesity paradox in heart failure. Can J Cardiol 31(2):195-202.

59. Pischon T, Boeing H, Hoffmann K, Bergmann M, Schulze MB, et al. (2008) General and abdominal adiposity and risk of death in Europe. N Engl J Med 359(20): 2105-2120.

60. Iliodromiti S, Celis Morales CA, Lyall DM, Anderson J, Gray SR, et al. (2018) The impact of confounding on the associations of different adiposity measures with the incidence of cardiovascular disease: A cohort study of 296535 adults of white European descent. Eur Heart J 39(17): 1514-1520.

61. Zheng R, Zhou D, Zhu Y (2016) The long-term prognosis of cardiovascular disease and all-cause mortality for metabolically healthy obesity: A systematic review and meta-analysis. J Epidemiol Community Health 70(10): 1024-1031.

62. Cirulli ET, Guo L, Leon Swisher C, Shah N, Huang L, et al. (2019) Profound perturbation of the metabolome in obesity is associated with health risk. Cell Metabolism 29(2): 488-500.

63. Kuk JL, Rotondi M, Sui X, Blair SN, Ardern CI (2018) Individuals with obesity but no other metabolic risk factors are not at significantly elevated all-cause mortality risk in men and women. Clin Obes 8(5): 305-312.

64. Bell JA, Carslake D, O’Keeffe LM, Frysz M, Howe LD, et al. (2018) Associations of body mass and fat indexes with cardiometabolic traits. J Am Coll Cardiol 72(24): 3142-3154.

65. Nazare JA, Smith J, Borel AL, Aschner P, Barter P, et al. (2015) Usefulness of measuring both body mass index and waist circumference for the estimation of visceral adiposity and related cardiometabolic risk profile (from the INSPIRE ME IAA study). Am J Cardiol 115(3): 307-315.

66. Bowman K, Atkins JL, Delgado J, Kos K, Kuchel GA, et al. (2017) Central adiposity and the overweight risk paradox in aging: Follow-up of 130,473 UK Biobank participants. Am J Clin Nutr 106(1): 130-135.

67. Gruberg L, Weissman NJ, Waksman R, Fuchs S, Deible R, et al. (2002) The impact of obesity on the short-term and long-term outcomes after percutaneous coronary intervention: the obesity paradox? J Am Coll Cardiol 39(4): 578-584.

68. Lin CF, Chen JW (2013) Obesity paradox-The controversial role of body mass index and plasma adiponectin in coronary artery disease and acute coronary syndrome. Acta Cardiol Sin 29(5): 381-386. 